\title{
The combined utilization of Chlorhexidine and Voriconazole or Natamycin to combat Fusarium infections
}

\author{
Tao Jiang ${ }^{1 \dagger}$, Jing Tang ${ }^{2 \dagger}$, Zhiqin $\mathrm{Wu}^{3}$, Yi Sun ${ }^{4}$, Jingwen $\operatorname{Tan}^{5^{*}}$ (1) and Lianjuan Yang ${ }^{5}$
}

\begin{abstract}
Background: Fusarium species are the fungal pathogens most commonly responsible for the mycotic keratitis, which are resistant to the majority of currently available antifungal agents. The present study was designed to assess the efficacy of a combination of low doses chlorhexidine with two other commonly used drugs (voriconazole and natamycin) to treat Fusarium infections.
\end{abstract}

Results: We utilized combinations of chlorhexidine and natamycin or voriconazole against 20 clinical Fusarium strains in vitro using a checkerboard-based microdilution strategy. In order to more fully understand the synergistic interactions between voriconazole and chlorhexidine, we utilized a Galleria mellonella model to confirm the combined antifungal efficacy of chlorhexidine and voriconazole in vivo. We found that for voriconazole, natamycin, and chlorhexidine as single agents, the minimum inhibitory concentration (MIC) ranges were 2-8, 4-16, and > $16 \mu \mathrm{g} / \mathrm{ml}$, respectively. In contrast, the MIC values for voriconazole and chlorhexidine were reduced to $0.25-1$ and $1-2 \mu \mathrm{g} / \mathrm{ml}$, respectively, when these agents were administered in combination, with synergy being observed for 90\% of tested Fusarium strains. Combined chlorhexidine and natamycin treatment, in contrast, exhibited synergistic activity for only $10 \%$ of tested Fusarium strains. We observed no evidence of antagonism. Our in vivo model results further confirmed the synergistic antifungal activity of chlorhexidine and voriconazole.

Conclusions: Our results offer novel evidence that voriconazole and chlorhexidine exhibit synergistic activity when used to suppress the growth of Fusarium spp., and these agents may thus offer value as a combination topical antifungal treatment strategy.

Keywords: Fusarium, Chlorhexidine, Voriconazole, Natamycin, Synergistic

\section{Background}

Keratomycosis is a form of fungal infection that can be challenging to treat, and that can result in permanent and severe vision damage when not adequately treated [1]. Fusarium species are the causative pathogens in between 36 and $67 \%$ of all traumatic keratitis cases, in

\footnotetext{
* Correspondence: cecilia88903@Tongji.edu.cn

${ }^{\dagger}$ Tao Jiang and Jing Tang contributed equally to this study and are joint first authors.

${ }^{5}$ Department of Medical Mycology, Shanghai Skin Disease Hospital, Tongji University School of Medicine, Shanghai 200443, China

Full list of author information is available at the end of the article
}

which $F$. oxysporum was the most frequently isolated species followed by $F$. solani [2]. Fusarium-related keratitis remains challenging to treat as these fungi are intrinsically resistant to most available antifungal agents. While advances in the standard treatment for keratitis have been developed in recent years, with natamycin (NAT) and voriconazole (VOR) being the current treatment agents of choice, further optimization of these therapeutic regimens is still warranted [3].

While previous studies have shown that NAT can be effective for the treatment of Fusarium infections, and $5 \%$ NAT is currently the first-line treatment for mycotic

(c) The Author(s). 2020 Open Access This article is licensed under a Creative Commons Attribution 4.0 International License, which permits use, sharing, adaptation, distribution and reproduction in any medium or format, as long as you give appropriate credit to the original author(s) and the source, provide a link to the Creative Commons licence, and indicate if changes were made. The images or other third party material in this article are included in the article's Creative Commons licence, unless indicated otherwise in a credit line to the material. If material is not included in the article's Creative Commons licence and your intended use is not permitted by statutory regulation or exceeds the permitted use, you will need to obtain permission directly from the copyright holder. To view a copy of this licence, visit http://creativecommons.org/licenses/by/4.0/ The Creative Commons Public Domain Dedication waiver (http://creativecommons.org/publicdomain/zero/1.0/) applies to the data made available in this article, unless otherwise stated in a credit line to the data. 
keratitis in certain nations, the poor penetration of this compound has been linked to failed treatment in some cases [1]. More recently, the application of $1 \%$ topical VOR has been shown to be an effective means of treating refractory fungal keratitis [4] while also exhibiting satisfactory diffusion within the aqueous humor. However, single-agent VOR treatment has not been shown to be adequately protective as a means of treating some patients, suggesting that combination therapies may be necessary to achieve reliable and durable therapeutic efficacy [5]. Chlorhexidine (CHL) is an antiseptic agent that is commonly used and has been shown to be safe for ophthalmic exposure at concentrations of $1 \%$ or below [6]. Furthermore, the intravitreal injection of $0.1 \%$ CHL has been shown to be a safe antiseptic strategy [7]. The utility of CHL for treating keratomycosis, however, remains to be established.

The goal of the present study was to assess the impact of combinations of NAT or VOR with CHL on clinical Fusarium isolates. We employed a checkerboard microdilution strategy to reliably identify potentially useful combinations of these therapeutic agents in vitro. We then employed a $G$. mellonella model to validate our findings in vivo.

\section{Results}

Assessment of the in vitro antifungal activity of CHL, NAT, and VOR

CHL, VOR, and NAT solutions exhibited Minimum inhibitory concentration (MIC) values of $>16 \mu \mathrm{g} / \mathrm{ml}, 2$ $8 \mu \mathrm{g} / \mathrm{ml}$, and $4-16 \mu \mathrm{g} / \mathrm{ml}$ to the Fusarium isolates, respectively. CHL did not exhibit antifungal activity, even at the highest tested concentration. When combined with VOR, the MIC values for CHL and VOR were reduced to $1-$ $2 \mu \mathrm{g} / \mathrm{ml}$ and $0.25-1 \mu \mathrm{g} / \mathrm{ml}$, respectively, with synergy being observed for 18 Fusarium strains (90\%). In contrast, such synergistic interactions were only observed for 2 Fusarium strains $(10 \%)$ treated with a combination of $\mathrm{CHL}$ and NAT (Table. 1). We did not observe any evidence of antagonism in any of these analyses.

\section{Assessment of the in vivo antifungal activity of $\mathrm{CHL}$ and VOR in G. mellonella}

In order to evaluate the synergistic efficacy of $\mathrm{CHL}$ and VOR in vivo, we infected G. mellonella with $F$. solani Jsfs1 and then treated these larvae using $\mathrm{CHL}$ and/or VOR. The survival in groups treated with VOR, CHL,

Table 1 Combination activity of CHL with VOR or NAT against Fusarium species

\begin{tabular}{|c|c|c|c|c|c|c|c|c|}
\hline \multirow[t]{2}{*}{ Strains } & \multirow[t]{2}{*}{ Origin } & \multicolumn{3}{|c|}{ MICs $(\mu \mathrm{g} / \mathrm{ml})$} & \multirow[b]{2}{*}{$\mathrm{FICl}$} & \multicolumn{2}{|c|}{ MICs $(\mu \mathrm{g} / \mathrm{ml})$} & \multirow[b]{2}{*}{$\mathrm{FICl}$} \\
\hline & & $\mathrm{CHL}$ & VOR & $\mathrm{CHLNOR}$ & & NAT & $\mathrm{CHL} / \mathrm{NAT}$ & \\
\hline \multicolumn{9}{|l|}{ F. Solani } \\
\hline Jzfs1 & Skin & $>16$ & 4 & $1 / 0.5$ & SYN & 8 & $2 / 8$ & $N$ \\
\hline Jzfs2 & Skin & $>16$ & 2 & $1 / 0.5$ & SYN & 8 & $2 / 8$ & $\mathrm{~N}$ \\
\hline Jzfs3 & Cornea & $>16$ & 2 & $1 / 1$ & $\mathrm{~N}$ & 8 & $1 / 8$ & $\mathrm{~N}$ \\
\hline Jzfs4 & Cornea & $>16$ & 8 & $2 / 0.5$ & SYN & 4 & $2 / 4$ & $\mathrm{~N}$ \\
\hline Jzfs5 & Skin & $>16$ & 2 & $2 / 0.5$ & SYN & 8 & $2 / 8$ & $\mathrm{~N}$ \\
\hline Jzfs6 & Cornea & $>16$ & 4 & $1 / 0.5$ & SYN & 16 & $2 / 4$ & SYN \\
\hline Jzfs7 & Skin & $>16$ & 4 & $1 / 0.5$ & SYN & 8 & $1 / 4$ & $\mathrm{~N}$ \\
\hline Jzfs8 & Auditory canal & $>16$ & 2 & $1 / 0.5$ & SYN & 8 & $1 / 4$ & $\mathrm{~N}$ \\
\hline Jzfs9 & Auditory canal & $>16$ & 2 & $1 / 0.5$ & SYN & 8 & $1 / 4$ & $N$ \\
\hline Jzfs10 & Nail & $>16$ & 2 & $2 / 0.5$ & SYN & 4 & $2 / 4$ & $\mathrm{~N}$ \\
\hline Jzfs11 & Nail & $>16$ & 2 & $1 / 0.5$ & SYN & 8 & $1 / 4$ & $\mathrm{~N}$ \\
\hline Jzfs 12 & Cornea & $>16$ & 4 & $1 / 0.5$ & SYN & 8 & $1 / 4$ & $\mathrm{~N}$ \\
\hline \multicolumn{9}{|c|}{ F. oxysporum } \\
\hline Jzfol & Cornea & $>16$ & 2 & $1 / 0.25$ & SYN & 8 & $2 / 4$ & $N$ \\
\hline Jzfo2 & Nail & $>16$ & 4 & $2 / 0.5$ & SYN & 8 & $1 / 8$ & $N$ \\
\hline Jzfo3 & Skin & $>16$ & 4 & $2 / 0.5$ & SYN & 8 & $1 / 2$ & SYN \\
\hline Jzfo4 & Skin & $>16$ & 2 & $1 / 1$ & $\mathrm{~N}$ & 8 & $2 / 8$ & $\mathrm{~N}$ \\
\hline Jzfo5 & Cornea & $>16$ & 2 & $1 / 0.25$ & SYN & 8 & $2 / 4$ & $\mathrm{~N}$ \\
\hline Jzfo6 & Nail & $>16$ & 2 & $1 / 0.5$ & SYN & 4 & $1 / 4$ & $N$ \\
\hline Jzfo7 & Auditory canal & $>16$ & 2 & $1 / 0.5$ & SYN & 8 & $1 / 4$ & $\mathrm{~N}$ \\
\hline Jzfo8 & Nail & $>16$ & 2 & $1 / 0.5$ & SYN & 8 & $1 / 4$ & $\mathrm{~N}$ \\
\hline
\end{tabular}

SYN synergy $(\mathrm{FICl} \leq 0.5), N$ indifference (no interaction $0.5<\mathrm{FICl} \leq 4), C H L$ Chlorhexidine, VOR Voriconazole, NAT Natamycin, MIC minimum inhibitory concentration, $\mathrm{FICl}$ Fractional inhibitory concentration index 
and VOR with CHL was $15 \%, 10 \%$ and $33.3 \%$, respectively. VOR treatment slightly improved larval survival, whereas $\mathrm{CHL}$ alone failed to improve larval survival, compared with the conidia group. Treatments with VOR combined with CHL significantly $(P<0.05)$ prolonged the survival of larvae (Fig. 1). Together, these in vivo findings thus confirmed the synergistic antifungal activity of CHL and VOR as evidenced by improved larval survival.

\section{Histopathological analyses}

On day 3 post- $F$. solani Jzfs1 infection, we conducted a histopathological assessment of larvae in this study. We observed the formation of $F$. solani spore and hyphae clusters in infected tissues regardless of treatment status (Fig. 2). There were 6, 4, 3 and 2 visible fungal clusters in the control group (Fig. 2a and e), CHL group (Fig. 2b and f), VOR group (Fig. 2c and g) and $\mathrm{CHL}+\mathrm{VOR}$ group (Fig. $2 \mathrm{~d}$ and $\mathrm{h}$ ), respectively. VOR treatment was associated with a slight reduction of the number of visible fungal clusters relative to control and CHL groups. The combination treatment group exhibited dramatic reductions of the number of visible fungal clusters relative to the other three evaluated groups.

\section{Discussion}

Herein we evaluated the potential combination antifungal activity of antifungal agents against pathogenic $\mathrm{Fu}$ sarium spp. CHL, which is a common, inexpensive, safe, and efficacious antiseptic agent exhibited promising performance. CHL is functions by binding to cell membranes and thereby impairing bacterial adhesion and driving the leakage of bacterial cellular contents [8]. Most bacterial and Candida species have been shown to be killed by $1-2 \%$ CHL solutions [9], while CHL concentrations of $1 \%$ or lower have been shown to be safe when used for ophthalmic purposes [10]. There are several studies showing that $\mathrm{CHL}$ exhibits in vitro antifungal activity against Fusarium spp. Oliveira et al. found that CHL exhibited fungicidal activity against $90 \%$ of tested F. oxysporum strains and $100 \%$ of tested F. solani strains when evaluating 98 strains isolated from fungal keratitis patients [11]. Xu et al. found that the MIC range for $\mathrm{CHL}$ is $8-32 \mu \mathrm{g} / \mathrm{mL}$, while the $\mathrm{MIC}_{90}$ value of chlorhexidine was $32 \mu \mathrm{g} / \mathrm{mL}$ for $24 \mathrm{~F}$. solani strains [12]. In the Netherlands between 2005 and 2016, 89 cases of Fusarium keratitis from 16 different hospitals were identified, and in vitro susceptibility testing indicated that chlorhexidine was active against Fusarium spp. with a MIC range of $8-32 \mathrm{mg} / \mathrm{L}$ for $F$. solani and $1-64 \mathrm{mg} / \mathrm{L}$ for $F$. oxysporum [5]. In our study, we found that CHL did not cause any detectable inhibition of Fusarium species when used as a single agent at the highest tested concentration (MIC $>16 \mu \mathrm{g} / \mathrm{mL}$ ). The difference between our findings and these prior studies may be attributable to the fact that relatively few fungal isolates were tested and that lower concentrations of CHL were employed herein.

In two blinded randomized trials by the same investigators, they observed patients of fungal keratitis treated with natamycin compared to $\mathrm{CHL}$ gluconate at various concentrations. Their results indicated that $0.2 \% \mathrm{CHL}$ yielded the best results $[13,14]$. However, the overall estimate of effect was uncertain [15]. Fungal keratitis caused by F. solani has been successfully treated with a combination of $0.02 \%$ CHL and AMB (Amphotericin B), underscoring the potential of $\mathrm{CHL}$ as an approach to the clinical management of fungal keratitis [16]. However, data regarding treatment with a combination of CHL and VOR is still limited.

In this study, we evaluated therapeutic interactions between CHL and VOR or NAT via a checkerboard microdilution strategy. As a first-line drug used for the management of fungal keratitis, NAT exhibited poor synergy ability with CHL both in vitro and in vivo. In

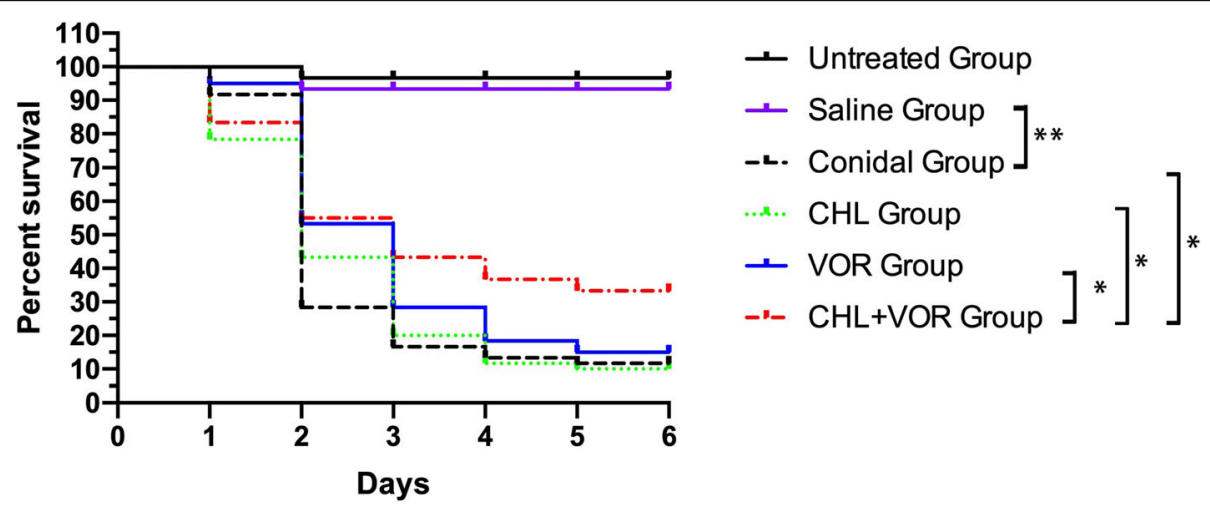

Fig. 1 G. mellonella survival rates. Untreated Group, wild type lava without Fusarium infection; Saline Group, wild type larvae injection with saline; Conidial Group, larvae infection with Fusarium without any treatment; CHL Group, Fusarium infected larvae treated with CHL only; VOR Group, Fusarium infected larvae treated with VOR only; CHL + VOR Group, Fusarium infected larvae treated with CHL combined with VOR; CHL: Chlorhexidine; VOR: Voriconazole; NAT: Natamycin. The experiment was repeated thrice on different days. *, $p<0.05$; ${ }^{* *} p<0.0001$ 


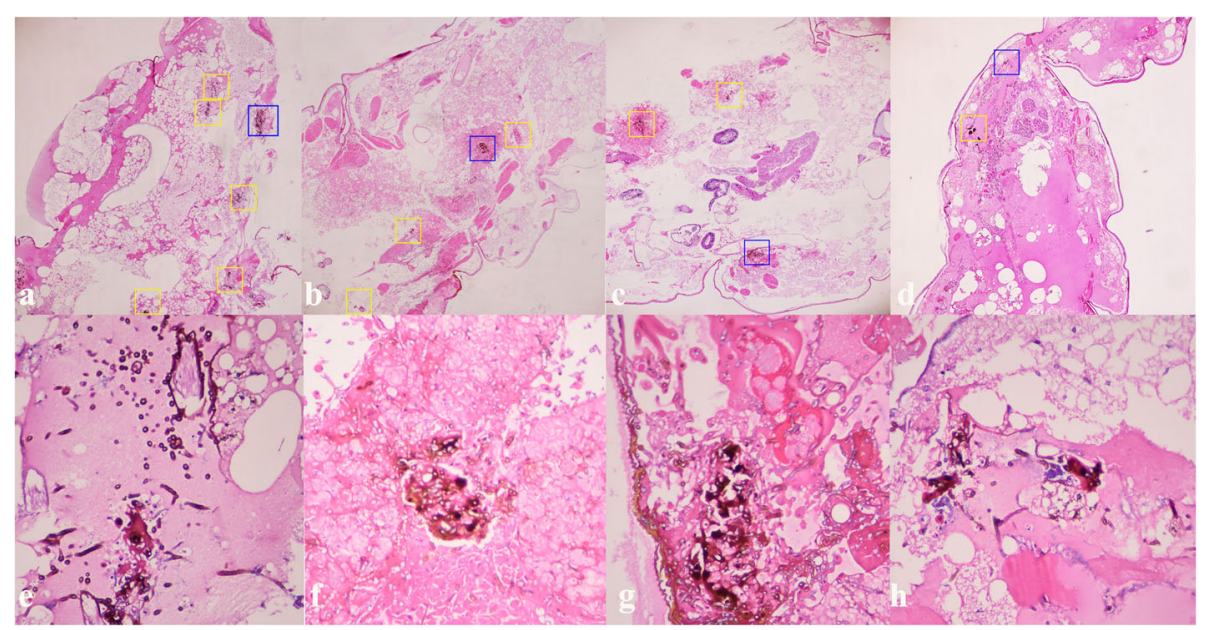

Fig. 2 G. mellonella histopathology. a-d, HE, 100x; e-h, HE, 400x. a and e, Saline treatment group; $\mathbf{b}$ and $\mathbf{f}$, CHL treatment group; c and $\mathbf{g}$, VOR treatment group; $\mathbf{d}$ and $\mathbf{h}, \mathrm{CHL}$ combine VOR treatment group; Yellow and blue frame, F. solani spore and hyphae clusters; Blue frame, The magnification part

contrast, the combination of CHL and VOR treatment exhibited synergistic activity against $90 \%$ of tested Fusarium strains. We observed no evidence of antagonism. As G. mellonella exhibit immunological response similar to those of mammals and are easy to manipulate, can be maintained at low costs, and incur minimal ethical concerns [17], they can be used as an ideal model system for studies of fungal virulence and antifungal drug activity [18]. We therefore utilized a G. mellonella model to evaluate the in vivo synergistic activity of these treatments. We determined that combination VOR + CHL treatment of infected G. mellonella larvae was associated with significant increases in larval survival. What's more, the concentration of CHL is about $0.00015 \%$, which is very low and proved to be safe when used in ophthalmic [10]. The mechanisms underlying this synergy are likely attributable to the ability of CHL to increase VOR penetration and/or to direct damage to Fusarium cell membranes without affecting drug efflux pump activity [19].

In order to expand upon these in vivo studies, we additionally conducted microscopic analyses of infected larvae (Fig. 2), which confirmed that combination treatment was associated with a reduction in the degree of tissue damage observed in G. mellonella larvae relative to control groups, suggesting that these two compounds exhibit excellent synergy in vitro and in vivo and are thus ideal for treating Fusarium keratitis.

\section{Conclusions}

Our results demonstrate that CHL and VOR exhibit synergistic efficacy against Fusarium species in vitro and in vivo. These findings suggest that $\mathrm{CHL}$ and VOR may be a viable therapeutic combination treatment for Fusarium infections, although future clinical trials and studies will be needed to validate this finding and to explore the underlying molecular mechanisms.

\section{Methods}

\section{Fungal strains}

In total, we obtained 20 clinical Fusarium isolates (12 F. solani and $8 F$. oxysporum strains) from clinical cultures (Table 1). These fungi were identified based upon a combination of morphological analyses and sequencing of the internal transcribed spacer (ITS) rDNA and translation elongation factor (TEF) $1 \alpha$ coding regions [20].

\section{Antifungal agents}

VOR (purity $\geq 99 \%$ ), NAT (purity $\geq 99 \%$ ) and CHL (purity $\geq 99 \%$ ) were obtained as powders from Selleck Chemicals (TX, USA), and were dissolved with DMSO (Amresco, OH, USA) to prepare $1600 \mu \mathrm{g} / \mathrm{mL}$ stock solutions.

\section{Inoculum preparation}

Fusarium strains were grown for 7 days at $30{ }^{\circ} \mathrm{C}$ on Sabouraud dextrose agar (SDA), after which they were isolated and resuspended in a $2 \mathrm{~mL}$ volume of sterile saline. Sterile gauze was utilized to filter conidia suspensions, after which a hemocytometer was used to quantify concentrations therein, which were adjusted to $1-5 \times 10^{6} \mathrm{cfu} / \mathrm{mL}$.

\section{Assessment of single-agent antifungal activity in vitro}

We conducted antifungal susceptibility tests based upon the CLSI M38-A2 [21]. Briefly, stock solutions were diluted in a two-fold serial manner using RPMI-1640 (Gibco, NY, USA) to yield final concentrations of $0.0313-16 \mu \mathrm{g} / \mathrm{ml}$. Microdilution wells were then filled with $100 \mu \mathrm{L}$ of appropriate Fusarium isolates at $1-5 \times$ $10^{4} \mathrm{cfu} / \mathrm{mL}$. Plates were incubated for $48 \mathrm{~h}$ at $35^{\circ} \mathrm{C}$, after which MIC values were determined by identifying the minimum antifungal agent dose necessary to achieve $100 \%$ inhibition of fungal growth relative to control 
untreated wells. We additionally included $A$. flavus strain ATCC 204304 as a quality control strain in the present analysis.

\section{Assessment of in vitro interactions between $\mathrm{CHL}$ and VOR or NAT}

A checkerboard microdilution strategy was used to evaluate synergistic interactions between CHL and NAT or VOR against Fusarium strains, with this approach having been adapted from the CLSI M38-A2 microdilution method. Briefly, we added $50 \mu \mathrm{l}$ volumes of seriallydiluted VOR or NAT horizontally across microdilution plates, while $50 \mu \mathrm{l}$ CHL samples that had been serially diluted were added in a vertical direction, with $100 \mu \mathrm{l}$ of a prepared inoculum suspension also being added to each well. Incubation times and MIC determinations for this assay were as above. Combination drug interactions were classified based upon the fractional inhibitory concentration index (FICI) [22] which was calculated as follows: $\mathrm{FICI}=(\mathrm{MIC} \mathrm{A}$ in combination/MIC $\mathrm{A}$ alone $)+$ (MIC B in combination/MIC B alone). Synergy was said to exist if FICI was $\leq 0.5$, while the interaction was said to be indifferent when FICI was $>0.5$ and $<4.0$, and antagonistic when FICI was $\geq 4.0$. Assays were conducted in duplicate on different days to ensure validity.

\section{G. mellonella survival assays}

Galleria mellonella caterpillars from the final instar larval developmental stage (Chengdu Pets and Insects Company, Sichuan, China) were maintained under dark conditions and were utilized within 1 week of receipt. In total, 20 randomly selected larvae $(330 \pm 25 \mathrm{mg}, 2-3 \mathrm{~cm})$ were utilized per group. Two control groups were injected with $10 \mu \mathrm{L}$ of saline or with no solution, respectively. Infected animals were injected with a $10 \mu \mathrm{l}$ volume containing Fusarium Jzfs1 $\left(1 \times 10^{7} / \mathrm{mL}\right)$ using a $25 \mu \mathrm{l}$ Hamilton syringe. Injections were made into the hemocoel of each larva through the last left proleg, with this region having first been cleaned with an alcohol swab. Following the completion of this injection process, larvae were transferred to plastic containers at $37^{\circ} \mathrm{C}$, with survival being assessed each day over a 6-day period. All experiments were conducted in triplicate.

\section{Assessment of VOR and CHL efficacy for the treatment of Fusarium infections alone or combination in G. mellonella} Galleria mellonella killing assays were conducted at $37^{\circ} \mathrm{C}$, as above, with $1 \times 10^{7} / \mathrm{ml}$ cells/larva being used for initial inoculation. VOR and CHL were diluted in saline and then used to treat infected G. mellonella in the following combinations: VOR $(3 \mu \mathrm{g} / \mathrm{mL}), \mathrm{CHL}(1.5 \mu \mathrm{g} /$ $\mathrm{mL})$, and $\mathrm{VOR}+\mathrm{CHL}(3 \mu \mathrm{g} / \mathrm{mL}$ and $1.5 \mu \mathrm{g} / \mathrm{mL}$, respectively). As controls, additional G. mellonella caterpillars were injected twice using saline. All drugs were injected through the last left proleg of each larva. The G. mellonella survival curves were analyzed by the Kaplan-Meier method, and differences were determined by using the log-rank (Mantel Cox) test. Differences were considered significant at $P$ values of $<0.05$.

\section{Histological analyses}

Fusarium presence within G. mellonella tissues was assessed via collecting larvae from each group on day 3 post-infection and treatment. These larvae were fixed with $10 \%$ neutral formalin, after which they were dehydrated with an ethanol gradient (70, 80, 90, 96, and $100 \%$ ethanol). Samples were then paraffin and xylene embedded, sliced to prepare $8 \mu \mathrm{m}$ sections, and stained with hematoxylin and eosin (HE). Samples were then evaluated via an FSX100 fluorescence microscope (Olympus, Tokyo, Japan) at $10 \times$ and $40 \times$. As controls, saline-injected larvae were also collected.

\section{Abbreviations}

MIC: Minimum inhibitory concentration; FICl: Fractional inhibitory concentration index; NAT: Natamycin; VOR: Voriconazole; CHL: Chlorhexidine; AMB: Amphotericin B; ITS: Internal transcribed spacer; TEF: Translation elongation factor

\section{Acknowledgements}

Not applicable.

Authors' contributions

$\mathrm{TJ}$ and $\mathrm{T}$ carried out the in vitro and in vivo antifungal experiment. ZW and TJ collection and analysis the experiment data. JWT designed, interpreted the experiment data and wrote the manuscript. YS and LY revised the manuscript critically for important content. All authors read and approved the final manuscript.

\section{Funding}

This work was supported by the Hubei Province Health and Family Planning Scientific Research Project (grant number WJ2018H178) and the Natural Science Foundation of Hubei Province (grant number 2019CFB567) to Yi Sun; Shanghai Municipal Commission of Health and Family Planning (grant number 201940476) to Lianjuan Yang.

\section{Availability of data and materials}

All data generated or analyzed during this study are included in this published article. Access to raw data can be acquired by connecting to the corresponding author via email.

Ethics approval and consent to participate

Not applicable.

Consent for publication

Not applicable.

Competing interests

The authors declare that they have no competing interests.

\section{Author details}

'Department of Clinical Laboratory, Jingzhou Central Hospital, The Second Clinical Medical College, Yangtze University, Jingzhou 434100, China. ${ }^{2}$ Department of Stomatology, Jingzhou Central Hospital, The Second Clinical Medical College, Yangtze University, Jingzhou 434100, China. ${ }^{3}$ Department of Ophthalmology, Jingzhou Central Hospital, The Second Clinical Medical College, Yangtze University, Jingzhou 434100, China. ${ }^{4}$ Department of Dermatology, Jingzhou Central Hospital, The Second Clinical Medical College, Yangtze University, Jingzhou 434100, China. ${ }^{5}$ Department of Medical Mycology, Shanghai Skin Disease Hospital, Tongji University School of Medicine, Shanghai 200443, China. 
Received: 6 June 2020 Accepted: 28 August 2020

Published online: 05 September 2020

\section{References}

1. Mahmoudi S, Masoomi A, Ahmadikia K, Tabatabaei SA, Soleimani M, Rezaie S, Ghahvechian H, Banafsheafshan A. Fungal keratitis: an overview of clinical and laboratory aspects. Mycoses. 2018;61 (12):916-30.

2. Fekih O, Haj Said O, Zgolli HM, Mabrouk S, Bakir K, Nacef L. Microbiologic profile of the mycosic absess on a reference center in Tunisia. Tunis Med. 2019;97(5):644-9.

3. Austin A, Lietman T, Rose-Nussbaumer J. Update on the Management of Infectious Keratitis. Ophthalmology. 2017;124(11):1678-89.

4. Sharma S, Das S, Virdi A, Fernandes M, Sahu SK, Kumar Koday N, Ali MH, Garg P, Motukupally SR. Re-appraisal of topical 1\% voriconazole and 5\% natamycin in the treatment of fungal keratitis in a randomised trial. $\mathrm{Br} J$ Ophthalmol. 2015;99(9):1190-5.

5. Oliveira Dos Santos C, Kolwijck E, van Rooij J, Stoutenbeek R, Visser N, Cheng YY, NTY S, Verweij PE, Eggink CA. Epidemiology and Clinical Management of Fusarium keratitis in the Netherlands, 2005-2016. Front Cell Infect Microbiol. 2020;10:133.

6. Oakley C, Allen P, Hooshmand J, BJT V. Pain and antisepsis after ocular administration of povidone-iodine versus chlorhexidine. Retina. 2018;38(10):2064-6.

7. Oakley $\mathrm{CL}$, Vote BJ. Aqueous chlorhexidine $(0.1 \%)$ is an effective alternative to povidone-iodine for intravitreal injection prophylaxis. Acta Ophthalmol. 2016;94(8):e808-9.

8. Letzelter J, Hill JB, Hacquebord J. An overview of skin antiseptics used in Orthopaedic surgery procedures. J Am Acad Orthop Surg. 2019;27(16):599-606.

9. Scheibler E, da Silva RM, Leite CE, Campos MM, Figueiredo MA, Salum FG, Cherubini K. Stability and efficacy of combined nystatin and chlorhexidine against suspensions and biofilms of Candida albicans. Arch Oral Biol. 2018:89:70-6.

10. Hamill MB, Osato MS, Wilhelmus KR. Experimental evaluation of chlorhexidine gluconate for ocular antisepsis. Antimicrob Agents Chemother. 1984;26(6):793-6.

11. Oliveira Dos Santos C, Kolwijck E, van der Lee HA, Tehupeiory-Kooreman MC, Al-Hatmi AMS, Matayan E, Burton MJ, Eggink CA, Verweij PE. In Vitro activity of Chlorhexidine compared with seven antifungal agents against 98 Fusarium isolates recovered from fungal keratitis patients. Antimicrob Agents Chemother. 2019;63(8):e02669-18.

12. Xu Y, He Y, Zhou L, Gao C, Sun S, Wang X, Pang G. Effects of contact lens solution disinfectants against filamentous fungi. Optom Vis Sci. 2014;91(12):1440-5.

13. Rahman MR, Minassian DC, Srinivasan M, Martin MJ, Johnson GJ. Trial of chlorhexidine gluconate for fungal comeal ulcers. Ophthalmic Epidemiol. 1997;4(3):141-9.

14. Rahman MR, Johnson GJ, Husain R, Howlader SA, Minassian DC. Randomised trial of $0.2 \%$ chlorhexidine gluconate and $2.5 \%$ natamycin for fungal keratitis in Bangladesh. Br J Ophthalmol. 1998;82(8):919-25.

15. FlorCruz NV, Evans JR. Medical interventions for fungal keratitis. Cochrane Database Syst Rev. 2015;4:CD004241.

16. Boral $\mathrm{H}$, van Diepeningen A, Erdem E, Yagmur M, de Hoog GS, Ilkit M, Meis JF, Al-Hatmi AMS. Mycotic keratitis caused by Fusarium solani sensu stricto (FSSC5): a case series. Mycopathologia. 2018;183(5):835-40.

17. Binder U, Maurer E, Lass-Flörl C. Galleria mellonella: an invertebrate model to study pathogenicity in correctly defined fungal species. Fungal Biol. 2016; 120(2):288-95.

18. Trevijano-Contador N, Zaragoza O. Immune response of Galleria mellonella against human fungal pathogens. J Fungi (Basel). 2018;5(1):3.

19. Teixeira Kl, Araújo PV, Sinisterra RD, Cortés ME. Chlorhexidine: betacyclodextrin inhibits yeast growth by extraction of ergosterol. Braz $\mathrm{J}$ Microbiol. 2012;43(2):810-8.

20. Salah $\mathrm{H}$, Al-Hatmi AM, Theelen B, Abukamar M, Hashim S, van Diepeningen AD, Lass-Florl C, Boekhout T, Almaslamani M, Taj-Aldeen SJ. Phylogenetic diversity of human pathogenic Fusarium and emergence of uncommon virulent species. J Inf Secur. 2015;71(6):658-66.

21. John $\mathrm{H}$. Reference method for broth dilution antifungal susceptibility testing of filamentous fungi, approved standard. M38-A2. Clin Lab Stand Inst. 2008;28(16):1-35.

22. Odds FC. Synergy, antagonism, and what the chequerboard puts between them. J Antimicrob Chemother. 2003;52(1):1-1.

\section{Publisher's Note}

Springer Nature remains neutral with regard to jurisdictional claims in published maps and institutional affiliations.

\section{Ready to submit your research? Choose BMC and benefit from:}

- fast, convenient online submission

- thorough peer review by experienced researchers in your field

- rapid publication on acceptance

- support for research data, including large and complex data types

- gold Open Access which fosters wider collaboration and increased citations

- maximum visibility for your research: over $100 \mathrm{M}$ website views per year

At BMC, research is always in progress.

Learn more biomedcentral.com/submissions 